Military Technical College

Kobry El-Kobba

Cairo, Egypt

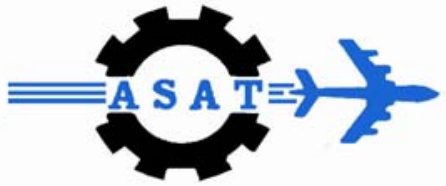

12-th International Conference

on

Aerospace Sciences \&

Aviation Technology

\title{
TRACTION BREAK SURFACE ANALYSIS OF THE AI-Zn COMPOSITE STRENGTHEN WITH CARBON FIBER
}

\author{
NEDELCU* D., COBZARU** ${ }^{* *}$.
}

\begin{abstract}
In this article are presented the results of the research about strengthening piece avulsion from the metallic matrix of $\mathrm{Al}$ and $\mathrm{Al}-50 \% \mathrm{Zn}$ that are obtained after the traction break. The results are legitimated about the aluminum carbide from the matrix- strengthening piece interface. Composites are obtained by liquid forging. And the strengthening piece is unidirectional aligned. The temperatures for the premolding preparation were about $20^{\circ} \mathrm{C}, 600^{\circ} \mathrm{C}$ and $900^{\circ} \mathrm{C}$. During the heating and the manipulations, it worked continually under the neuter argon atmosphere to protect these highly reactivity oxygen fibers. Measurements were made on testpieces tear to pieces by traction break into longitudinal sense of strengthening piece. After the test, surface was covered with a silver layer, witch was dreg from steam phase. Fibers were included into a transparent resin and after the splitting extraction length measurement was made. In this paper are presented fibers avulsions from aluminum matrix (maximum quantity of aluminum carbide from strengthening piece interface have the result of an strong adhesion and therefore fibers will tear into matrix breaking section plan) respectively $50 \% \mathrm{Zn}$ alloy aluminum matrix (Aluminum carbide cause an medium adhesion into interface and fibers tears into pieces according with histogram). Also are presented SEM microscopy of the Al matrix samples and $50 \% \mathrm{Zn}$ alloy aluminum matrix.
\end{abstract}

\section{KEY WORDS}

Traction, Al-Zn composite, carbon fiber

\footnotetext{
"Associate professor Ph.D., Technical University "Gh. Asachi" of lasi Romania; Email: dnedelcu@tcm.tuiasi.ro

"Lecturer Ph.D., Technical University "Stefan cel Mare" of Suceava Romania: Email: cobzaru@fim.usv.ro
} 


\section{GENERAL CONSIDERATIONS}

The composite materials can be defined according to the nature of their components (metal, carbon, glass, plastic, ceramics...), or according to the performances often related to density (largely spread or of high performances).

The advantages of this new class of materials are multiple and can be sum up as follows: unexpected possibilities of adapting the materials according to the functions of the pieces; possible optimizations of the weight, efforts, technological solutions that are based on: the selection of the materials and of their components; the geometrical configuration; the new transformation processes; obtaining new performances: reduced weight without interventions on properties; specific mechanical resistance (traction, fatigue...); chemical and electrical resistance adaptable to requirements; the diminution of production costs on unitary volume: direct costs (fabrication costs, energetic consumption...); indirect costs (related to life duration); possible adaptations between choosing the material, the amount of production and the manufacturing process.

The above mentioned are the basis of a new concept of "adaptable materials" that respond adequately to the field of imposed efforts (independent or combined). With their complex structure (easy and ductile matrix, reinforced with crack fibers that have very high characteristics), they lead to high performances with numerous opportunities in various disciplines.

The fibers are a volumes broken number often contained between $30-70 \%$ of the composite material (the ratio between the volume of the fibers and the total volume of the composite).

Two big categories can be distinguished: high performance fibers: these are glass, carbon fibers. There are also a few synthetic fibers with excellent thermal properties that are the object of a recent research; fibers with particular uses: these are fibers initially used as mechanic strengthening (before the development of the high performance fibers) for special applications: for example for thermal coatings, electric insulators, decorative elements, etc. They can be of natural origin (wood, cotton, paper...), synthetic (polyamide, polyester...) or metallic.

The adjoining between a matrix and the reinforced element can not be occasional. It depends on: the chemical compatibility of the materials in contact that influences the transmission of efforts through the interface; the technology of obtaining is chosen according to the geometry of the piece and the size of the production; the desired chemical, mechanic resistance; the cost of fabrication, of the basic products, of transformations and eventually of finishing.

The methods of dimensioning are made by using a computer with the following specifications: defining the appropriate geometry is based on: looking for a constant thickness, the orientation of the strengthening in the presupposed meaning of the main efforts, the identification of the areas with a strong contraction, geometrical particularities; choosing the degree of strengthening and packing (size and orientation), necessary to determine the mechanic characteristics; using the advanced methods of calculations, finite element type.

The presence of the strengthening raises considerably the elastic limit of the matrix, fact due to some more effects: the transfer of force to the strengthening, this diminishing the level of efforts in the matrix; a very low size of corn in the matrix, because of the interlock of the recrystallization and of the bulk up of the corns during the strengthening step; a very high level of cold -hardening. 
The break of a piece is the result of a succession of two phenomena: damage, which consists in the formation of defects (cavities, micro crazes) inside or at the surface of the piece; cleft, which consists of the propagation of one of these defects all the length of the piece.

For the pieces in the aeronautical industry that are checked during regular inspections with means and methods of non-destructive control, resistance to cleft determines the maximum admitted dimension of the defects detected. Mainly it is about the weak point of these materials and in conclusion about the main obstacle in extending the applications. A low adherence at interface (matrix $\mathrm{Al}-\mathrm{Zn}$ reinforced with carbon fibers) leads to big dimensions fibers pulled out, while a high adherence at interface causes the breaking of the fibers close to the plan of breaking the matrix. This effect was transformed in quantitative terms to estimate the influences of the matrix composition on the adherence at interface.

In the composites with aligned fibers, the pulling out of the fibers with the decreasing of the binding at interface leads, in a microscopic scale, to the increasing of the roughness of that surface after the test of traction in the longitudinal direction of the strengthening.

\section{RESEARCHES AND RESULTS}

After some more unsuccessful tests of obtaining the $A 1 / C$ composites by a continual casting under pressure, I adopted the technology of the liquid forging. The reduced number of mobile components of the installation and the rigorous temperature control guaranteed a good infiltration and a reduced accident risk. Being known the very low wetting of the carbon fibers by the liquid aluminum at actual temperatures when casting it was necessary the necessity of high fluidity of the matrix. A complete infiltration of the pre-form of carbon fibers is possible by increasing the temperature over 1000 degrees Celsius or the decreasing of this one and the increasing of the infiltration pressure at $20 \mathrm{MPa}$.

In order to find the optimal domain of the infiltration temperatures, at the constant pressure of $20 \mathrm{MPa}$, I performed a series of technological tests that lead me to the conclusion that the infiltration temperature has to be higher than 150 degrees Celsius over the liquids line from the equilibrium diagram. At low temperatures of infiltration and preparation of the pre form, the carbon fibers subside and do not allow because of a reduced wetting and fluidity a complete filling with no pores. The surfaces in movement were coated with paint made of dolomite in industrial alcohol.

After having established the domain of optimal temperatures for a complete infiltration, by the experiences held, I analyzed the evolution of the reaction in the interface, the role of the zinc alloying of the aluminum matrix and the influence of the heat treatment on the start of aluminum carbide in interface.

The temperatures of preparation of the pre form were of $20^{\circ} \mathrm{C}, 600^{\circ} \mathrm{C}$ and $900^{\circ}$ degrees Celsius. In order to protect these fibers of the high reactivity of the oxygen in the atmosphere, while heating and manipulating, work was done under a neutral atmosphere of argon.

In order to examine the role of zinc alloying, the aluminum matrix was modified with variable percentages of zinc. The used fibers are produced by TORAYKA firm which commercial characteristics are published in commercial leaflets, but many data are firm secrets and protected by very expensive patents. 
Using the Cartesian system of coordination, with the axis $Z$ along the fibers (meaning perpendicular on the surface of breaking), we can measure (as one can see in Fig.1 and Fig.2) the distribution of the values $A z$ for separate points at surface. The maximum of the value $\mathrm{Dz}$ modifies when increasing the $\mathrm{Zn}$ content of the matrix. This method offers a quantitative criterion of comparing the adherence at surface foe a series of similar composites.

The measurements were made on tubes broken by traction in the longitudinal direction of the strengthening. After the breaking, the surface was coverer with a silver layer deposit during the vapor phase. The fibers were included in a transparent resin and after the division into sections measurements of the length of extraction were made.

In Fig. 3 and Fig.4 are presented the SEM microscopy of the sample with A1 matrix respectively alloyed aluminum with $50 \%$ zinc.

\section{CONCLUSIONS}

The optimal smelting methods have to realize a compromise between:

-the use of strengthening with a high module of elasticity but less crack;

-the increasing of the adherence at interface without obtaining crack compounds.

For the same working conditions I obtained reproducible results with a distribution of the values in a very limited range;

-the layer of aluminum carbide in the interface changes radically the behavior of the composites in field effort;

-the fibers extracted from the matrix with a $100 \%$ Al have the minimum length and the medium plan of breaking almost can be confused with the plan of breaking of the matrix (fig.3);

-for the tubes from the second lot where the matrix has $50 \%$ zinc one can observe big lengths of fibers extracted broken on sections as far as possible from the plan the matrix broke. One can also remark the surface of the fibers that is completely unattached by the liquid matrix during the infiltration.

\section{REFERENCES}

[1] Thornton, P.H., Energy Absorption in Composite Structure, Journal of Composite Materials, Vol.13, July, (1979).

[2] Foye, R.L., Swindlehurst, C.W., Hodges, W.T., A Crashworthiness Test for Composite Fuselage Structure, Fibrous Composite in Structural Design, eds. Plenum Press, New York, p.241-258, (1980).

[3] Foye, R.L., Hodges, W.T. Some Results from a Crash Energy Absorption Test for Evaluating Composite Fuselage Construction, $37^{\text {th }}$ Annual Forum of the American Helicopter Society, May (1981).

[4] Timoshenko, N., Goodier, A., Mechanical Behavior of Metal Matrix Composites, edited by Cornie, J.A. and Crossman, F.W. TMS-AIME, Chicago, III, p. 319, (1979).

[5] Cobzaru, P., Delannay, F., Fenomene ce apar la interfața materialelor compozite cu matrice de $\mathrm{Al}, \mathrm{Cu}, \mathrm{Zn}$ ranforsată cu fibre continui, Cong. Mater. Sci., Montreal, (1993). 
Al $100 \%$

$(\mathrm{mm})$

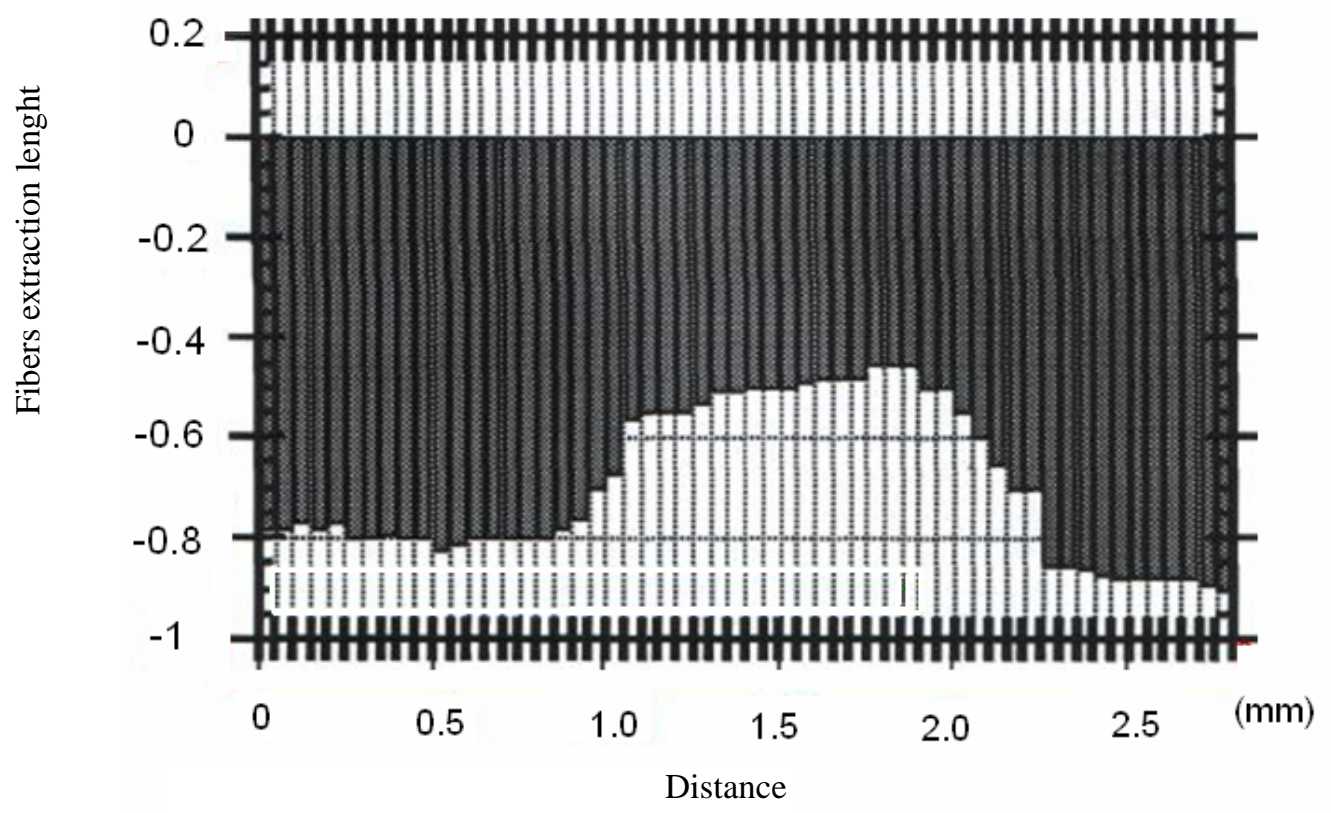

Fig.1. Pulling out of fibers from the aluminum matrix. The maximum quantity of aluminum carbide leads to a high adherence and in conclusion the fibers break in the plan of breaking of the matrix

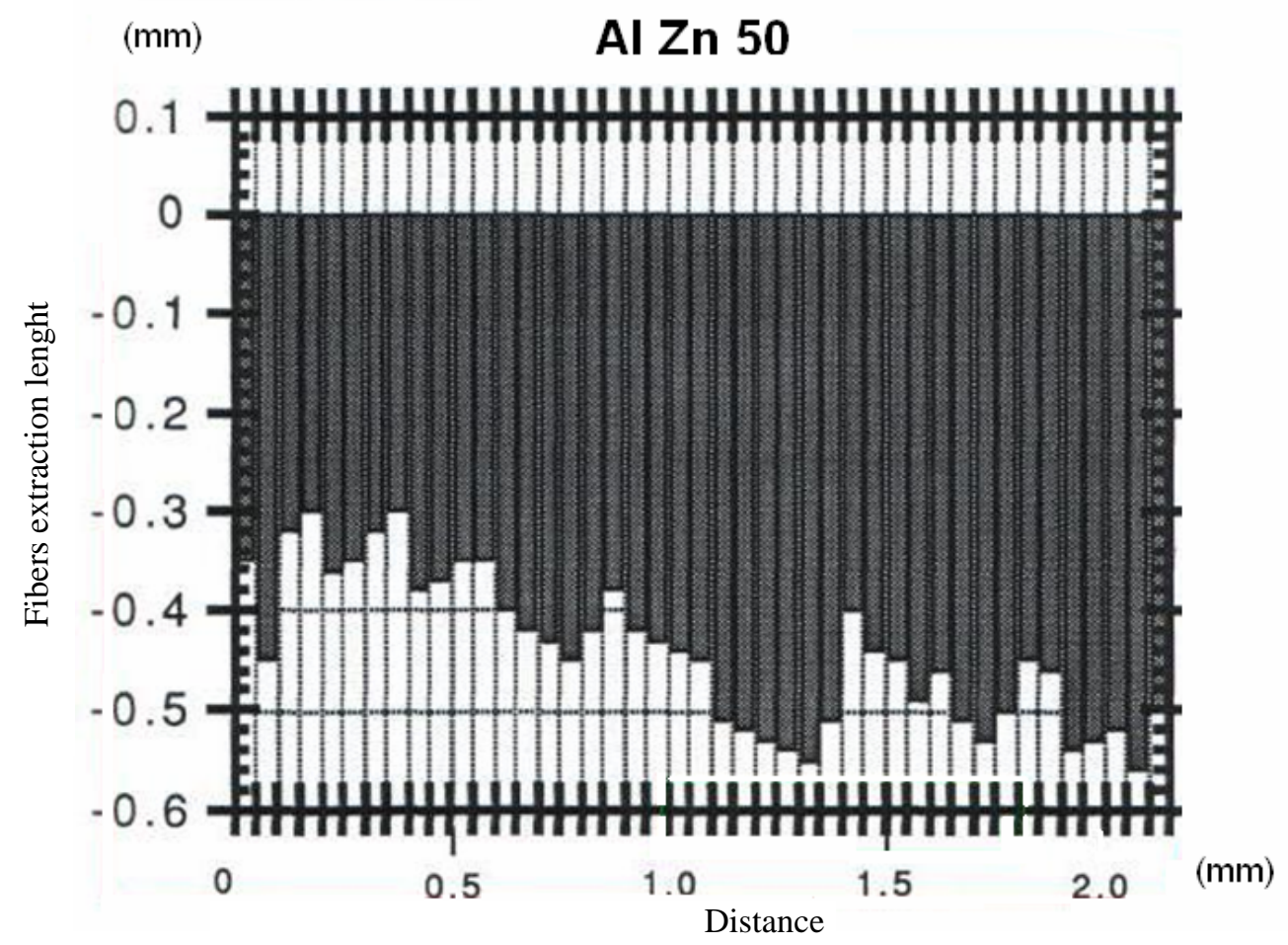

Fig.2. Pulling out of fibers from an aluminum matrix alloyed with $50 \%$ zinc. The aluminum carbide induces a medium adherence in interface and the fibers break as shown in the bar chart 


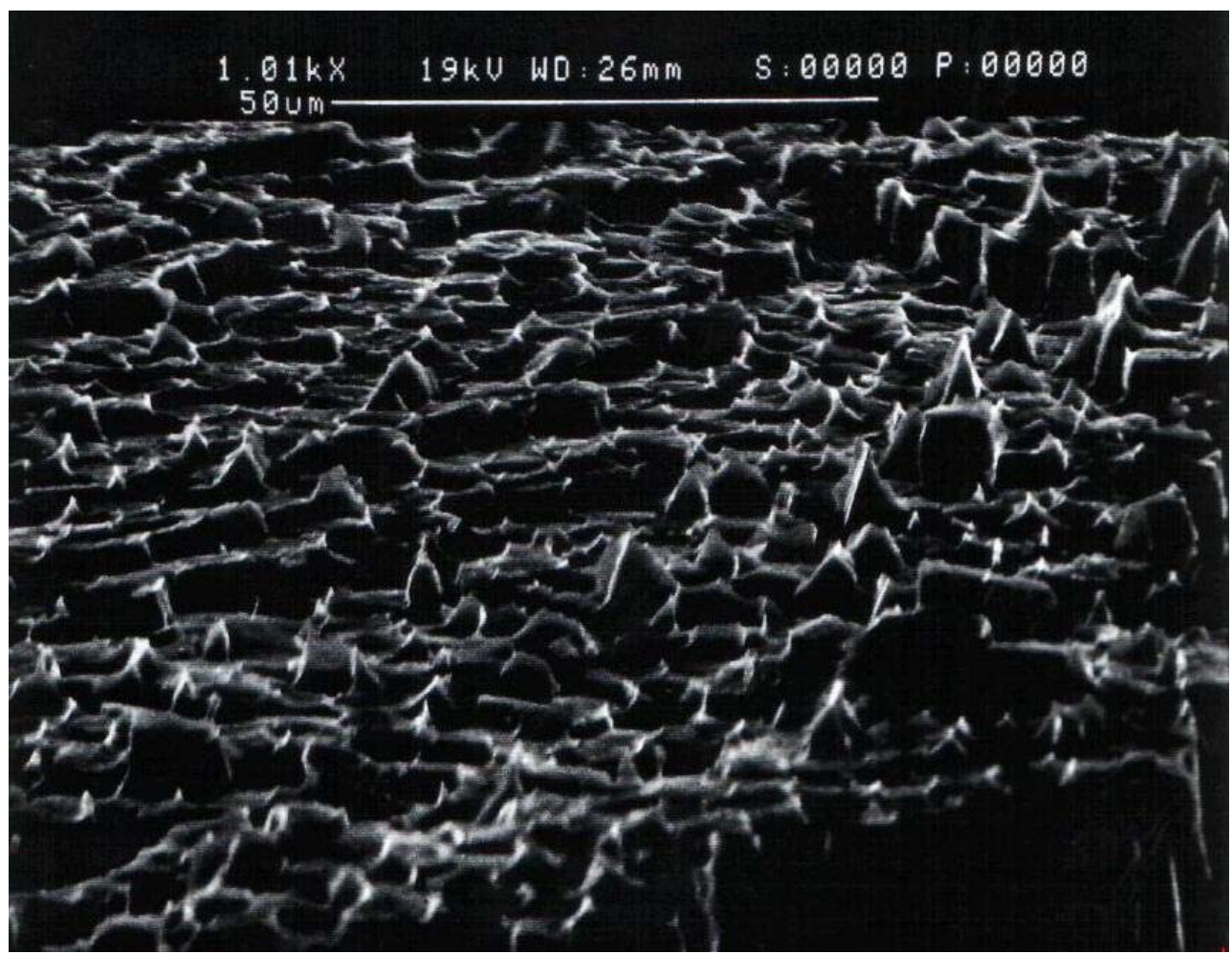

Fig.3. SEM microscopy of a composite with a $100 \%$ aluminum matrix, it can be seen the reduced length of the extracted fibers

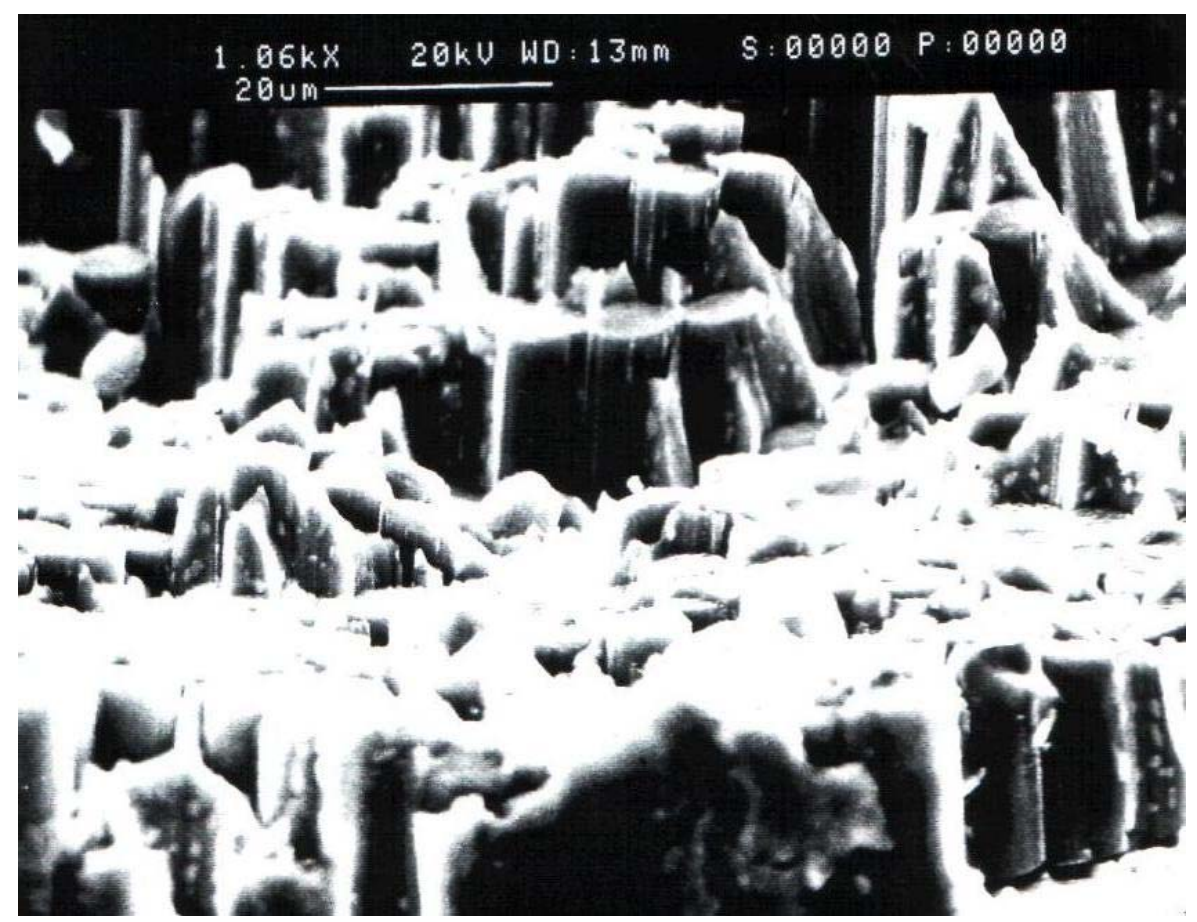

Fig.4. SEM microscopy of a composite with aluminum matrix alloyed with $50 \%$ zinc. The length of the extracted fibers can be evaluated by using the ruler above the image 